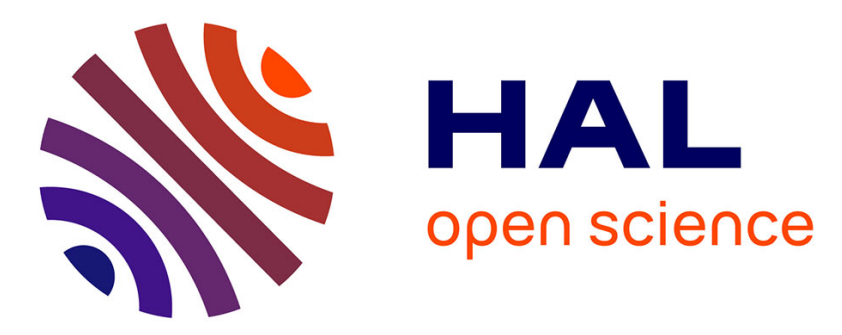

\title{
A low-cost multitouch spherical display: hardware and software design
}

Thomas Crespel, Patrick Reuter, Xavier Granier

\section{To cite this version:}

Thomas Crespel, Patrick Reuter, Xavier Granier. A low-cost multitouch spherical display: hardware and software design. SID's Digest of Technical Papers, 2017, Display Week 2017, 48 (1), pp.619-622. 10.1002/sdtp.11716 . hal-01455523

\section{HAL Id: hal-01455523 \\ https://hal.inria.fr/hal-01455523}

Submitted on 4 Jul 2018

HAL is a multi-disciplinary open access archive for the deposit and dissemination of scientific research documents, whether they are published or not. The documents may come from teaching and research institutions in France or abroad, or from public or private research centers.
L'archive ouverte pluridisciplinaire HAL, est destinée au dépôt et à la diffusion de documents scientifiques de niveau recherche, publiés ou non, émanant des établissements d'enseignement et de recherche français ou étrangers, des laboratoires publics ou privés. 


\section{A low-cost multitouch spherical display: hardware and software design}

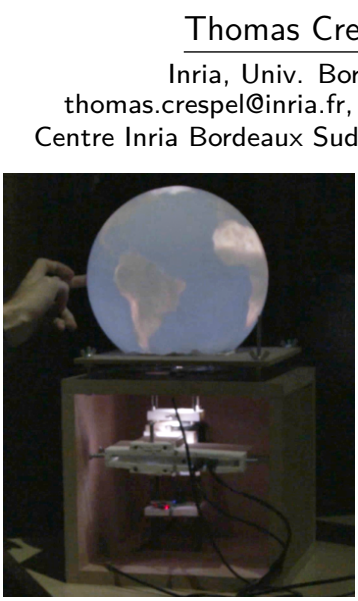

(a)
Patrick Reuter

Univ. Bordeaux/LaBRI, Inria patrick.reuter@u-bordeaux.fr

\section{Xavier Granier}

Institut d'Optique GS/LP2N, Inria xavier.granier@institutoptique.fr

thomas.crespel@inria.fr, Ph.D. student de la Vieille Tour - 33405 Talence Cedex - France / +33 (0)5 24574000

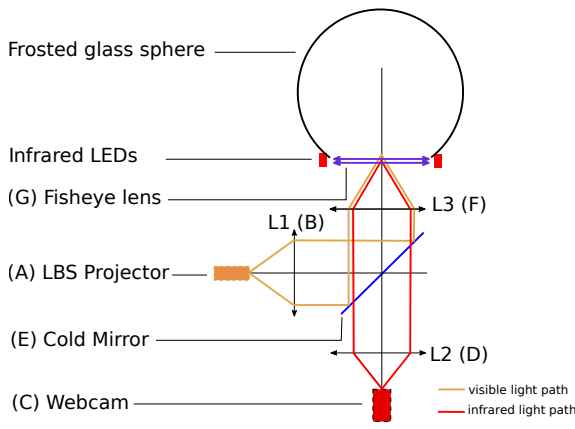

(b)

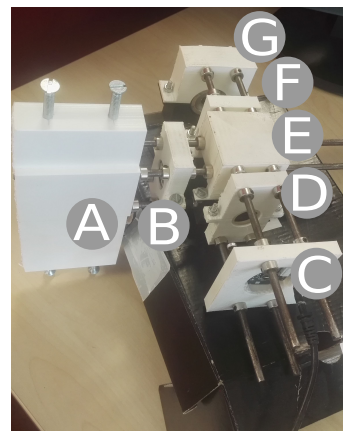

(c)

Figure 1: (a) An interactive globe on our spherical multitouch display, (b) outline of the optical system, and (c) its picture

\section{Abstract}

Spherical mulitouch displays offer exciting possibilities but are still costly. In this paper, we describe hardware and software considerations to build a more affordable one, with off-the-shelf optical components and 3D printed elements. We exploit the technology of laser-beam steering projectors and use optical tracking for multitouch.

\section{Objectives}

Touchable displays are becoming more and more part of our daily life, for the general public (smartphones, tablets) as well as for professionals (meeting tables, advertisement and information displays). Today, most of these interactive displays are flat, but there is a growing interest in designing curved displays, for example with OLED technology. However most of them are limited to developable shapes.

We define a spherical multitouch display as an opaque sphere, on which information is displayed and can be interacted with directly by touch. This kind of display has numerous applications, such as being used as a teaching support for mathematics or geography, as an original communication support, or as an ingenious tool for collaborative work or data visualization.

Spherical displays have not yet entered in every classroom, most probably due to the high cost of existing products, and so they remain non-conventional: users and developers are not necessarily familiar with. A whole new field begins to be studied in terms of interaction on spherical displays $[1,8,13]$, but a lot of work still needs to be done, and specific development environments have to be set up.

In this paper, we propose a new design of a low-cost, yet effective, spherical multitouch display. The principal components are a laser beam steering projector, a fisheye lens, a webcam for optical tracking, off-the-shelf optical components, and custom 3D printed parts, as well as software for tracking, calibration, and color correction. Our work makes multitouch spherical displays accessible to a larger number of people, so that any researcher or enthusiast interested in this field can contribute with hardware or software improvements, as well as user interaction studies.

The paper is organized as follows. In Section 2, we show previous work on spherical displays. In Section 3, we present the hardware and software components of our design and our prototype. Finally, in Section 4, we conclude and provide perspectives for further improvement.

\section{Background}

Global Imagination was the first company to patent and commercialize spherical displays that are not touchable [11]. Their product, the Magic Planet, is based on an optical coupling between a DLP projector and a fisheye lens that is specifically designed to conjugate the image plane of the projector onto the surface of the sphere.

ArcScience also sells non-touchable spherical displays following a different approach: they use a patented hemispherical convex mirror [5] to project the beam of the projector onto the inside of the sphere. The projection is done from the south pole of the sphere, and the hemispherical mirror is located at the north pole. The sphere is not fully covered because of casted shadows at the north pole.

To our knowledge, the first work on developing touchable spherical displays was presented by Microsoft Research [2]. In particular, they added multitouch capabilities to the spherical display from Global Imagination by using an infrared camera for optical finger tracking from the inside of the sphere, and a cold mirror to separate the injected infrared light from the visible light of the projector.

A commercially available multitouch spherical display is the PufferSphere, sold by Pufferfish [6]. The projection part relies, like previous ones, on the association of a DLP projector and a specially designed patented fisheye lens. The method to make it touchable, however, is not communicated by the company.

All of the examples above rely on tailored optics that are difficult to design and expensive to produce. Alternatively, Patel [9] proposed a low-cost and «Do it yourself» spherical display, however, it is not touchable. It exploits the technology of a focus-free laser beam steering projector which allows the projection of a sharp image on curved surfaces. The projector is associated with a commercially available afocal fisheye lens that allows to cover almost the entire surface of the sphere. 


\section{Results}

\subsection{Overview}

The main contribution of our work is the design of a spherical display with multitouch capabilities that is low-cost by using only off-the shelf optical components. Our hardware design is illustrated in Figure 1(b) and detailed in Section 3.2. For the display, we use a laser beam steering projector (LBS) combined with a standard fisheye lens to project the image on the interior surface of a frosted-glass sphere with an opening neck, as inspired by Patel [9]. For multitouch capabilities, we use optical tracking on a second light path with an infrared camera that senses the reflection of rear-projected LED infrared light, inspired by [2], but without requiring a custom shaped lens. We combine both light paths with a cold mirror, and we change the FOV of the projector and the camera with a lens system in order to reach almost $180^{\circ}$ at the output of the fisheye lens. Our lens system is designed to maintain the «focus-free» property of the projector through the optical system.

The finger tracking (Section 3.3) is done by analyzing the image of the infrared camera. Since we use standard optical components, we rely on calibrations (Section 3.4) for the projection, the tracking and the correction of chromatic aberrations. Other considerations on the prototype, together with its cost, are detailed in Section 3.5.

\subsection{Hardware components}

Projector For a spherical display, we need to have a projection system that is in focus everywhere on a spherical surface. Classical projectors do not allow such a property due to their limited depth of field, unless custom optics are used as in $[2,5,6,11]$. Consequently, we use a laser beam steering ( $L B S$ ) projector. LBS projectors have recently been introduced into the consumer market. They are focus-free, meaning that the projected image is always in focus and sharp everywhere, even on a curved surface, without the need of tailored optics. The focus-free property comes from its laser-based light source: three laser diodes (red, green and blue) are combined to a single, nearly collimated beam that rasters the space to construct the image pixel by pixel by using a scanning micromirror. The low divergence of the beam leads to a linear behavior of the pixel size along the projection distance.

We have choosen the Celluon PicoPro projector. It has an equivalent resolution of $1920 \times 720$, and its field of view (FOV) is $43^{\circ}$ horizontally and $22^{\circ}$ vertically. It is one of today's brightest LBS projectors available with 30 lumens, which is still quite low compared to for example DLP projectors. However, the contrast ratio is far better, and the colors are more vivid. We expect that the brightness of LBS projectors continues to grow in the future.

Fisheye lens The field of view of the projector is not wide enough to cover the entire inside surface of a sphere, so we use a fisheye lens to increase the projection angle up to nearly $\pm 90^{\circ}$. We use an afocal fisheye lens, which means that in contrast to fisheye objectives, it does not conjugate the object on an image plane but multiplies the angles by the magnification. The intended use of the lens is to be mounted on a camera so that the light coming from wide angles narrows towards the camera lens. In our work, we use the lens not only for this purpose, but also for the exact opposite: we want to expand the beam of the projector from small angles to reach nearly $\pm 90^{\circ}$ in the output. We decided to use a x0.2 magnification Opteka Fisheye lens, similar to Patel [9]. For the projection, the relevant magnification of this lens is $\mathrm{x} 5$, and so it requires an input angle of $\pm 18^{\circ}$ to achieve the $\pm 90^{\circ}$ output.

Infrared camera and illumination In order to make the spherical display touchable, we use optical tracking of the user's rear illuminated fingers, similar to Benko et al. [2]. Note that the camera for the tracking must work on the same optical axis as the projector to be able to use the fisheye lens, but in a different spectral range so that the displayed image does not interfere with the tracking. For the illumination, we have built a circular infrared LED ring operating at $880 \mathrm{~nm}$, which is a suitable wavelength since it is far enough from the maximum wavelength of the projector, but close enough to the visible spectrum in order to still be sensed by affordable cameras. Technically, we use 16 OSRAM SFH485 LEDs distributed circularly around the opening neck of the sphere, in order to achieve an as-uniform-as-possible illumination (see Figure 3). For convenience and mobility, the electric circuit is USB powered.

We use a PlayStation Eye camera, since it is a cheap and effective webcam, and its sensor can work in the near infrared range. This camera is commonly used for infrared tracking and can be modified with existing adapted material (e.g. [10]). We have replaced the original IR-cut filter located between the lens and the sensor by a bandpass filter centered on $880 \mathrm{~nm}$. We also modified the FOV by changing the main lens of the camera. The new lens has a focal length of $6 \mathrm{~mm}$ to obtain a field of view of $30^{\circ}$ horizontally and $23^{\circ}$ vertically. This makes the camera's FOV closer to the projector's FOV, and so they can share common lenses. Finally, we removed the case of the webcam to reduce useless volumes.

Lenses In our optical system illustrated in Figure 1(b), the micro-mirror of the projector is located at the focal point of L1 in order to collimate the projector's beam. The beam is then reflected on the cold mirror towards L3 that makes it converge with the desired input angle $\alpha$ on the fisheye lens. The angle $\alpha$ depends on the height $H_{3}$ of the beam on L3 and its focal length $f_{3}^{\prime}$, such that $\tan (\alpha)=H_{3} / f_{3}^{\prime}$. Assuming that the diameter of the lens is not limiting, $H_{3}$ is defined on the first lens as $H_{3}=H_{1}=f_{1}^{\prime} \tan (F O V / 2)$. According to the previous equations, the following setup results in the full cover of the sphere for projection and imaging:

- L1: $f_{1}^{\prime}=43 \mathrm{~mm}, D_{1}=23.5 \mathrm{~mm}$

- L2: $f_{2}^{\prime}=43 \mathrm{~mm}, D_{2}=23.5 \mathrm{~mm}$

- L3: $f_{3}^{\prime}=22 \mathrm{~mm}, D_{3}=18 \mathrm{~mm}$

Theoretically, the focal lens of L 2 could be $f_{2}^{\prime}=27.3 \mathrm{~mm}$, so that the input angle at the fisheye would be $\pm 18^{\circ}$ resulting in $\pm 90^{\circ}$ at the output. However, as a single degree of error in the prototype results in a 5 degree loss on the sphere, we have chosen the effective L2 lens to have a focal length of $22 \mathrm{~mm}$, so that up to $\pm 3^{\circ}$ of error are acceptable while keeping the full coverage.

Another important issue to take into consideration with an LBS projector is the evolution of the scanning laser beam diameter: the image is sharp since the beam has low divergence. Adding a lens makes the beam more diverging, resulting in a more blurry image. The second lens must put the waist near its focal point in order to restore back the low-divergence of beam (see Figure 2) and to make the image sharp again.

Cold mirror We use a cold mirror to differentiate the infrared illumination used for tracking from the visible light of the projector. More precisely, we use a $45^{\circ}$ cold mirror from KnightOptical: it is reflective in the visible spectrum (400$700 \mathrm{~nm})$ and transparent in the infrared spectrum $(>700 \mathrm{~nm})$. 


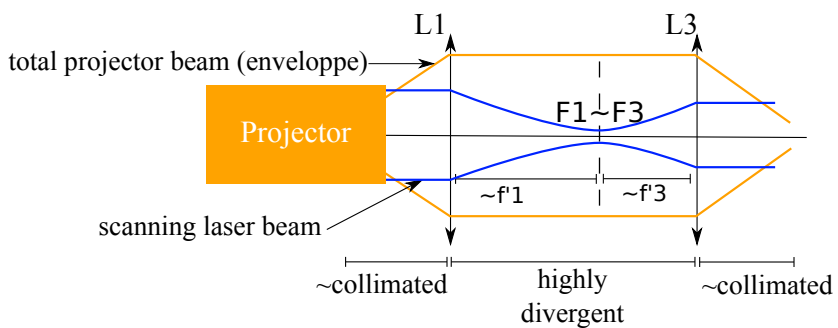

Figure 2: Evolution of the scanning laser beam diameter (not in scale) with the distance. The quasi-collimated laser of the projector is focused at F1 before diverging a lot. L3 makes the beam collimated again if the waist is also on its focal point.

If L1 is completely lit, the size of the collimated beam is approximately $17.5 \mathrm{~mm}$. The mirror we have chosen has a size of $40 \mathrm{~mm} \times 25 \mathrm{~mm}$ corresponding to an apparent surface at $45^{\circ}$ of $28.3 \mathrm{~mm} \times 25 \mathrm{~mm}$, so that the mirror is not a limiting optical element in the system.

\subsection{Finger Tracking}

We use optical tracking for the multitouch capability: the entire inside surface of the sphere is imaged onto the sensor of the infrared webcam, and then the image is processed in order to detect the position of the fingers. We use rear diffused illumination: the infrared light coming from the LED ring is partly reflected by the frosted glass sphere, and partly transmitted. When the user's finger points on the sphere, the transmitted infrared light is reflected by the fingers towards the webcam.

For finger tracking, the user has to set up a background image where no fingers are visible (Figure 3(a)). Then, in each frame, the current image (Figure $3(\mathrm{~b})$ ) is subtracted from the background before being binarized (Figure 3(c)) by an adjustable threshold in order to find the position of the fingers by segmentation. A series of intermediate filters increases the robustness, such as high frequency filtering and amplification. Despite the low difference of intensity with the quite intense background, the fingers are correctly detected. In Section 4, we discuss future improvements.

We use the third-party open source software Community Core Vision (CCV), developed by the NuiGroup community [7]. Among its advantages, its has a graphical user interface for adjusting the image processing parameters, and the tracking results are sent over a local network with the TUIO (Tangible User Interface Objects) protocol [4], a specific UDP protocol. These tracking results are all the relevant informations of the detected fingers, such as their ID, size, position and speed, as well as events such as adding, moving or removing a finger. This information can then be used to identify touch gestures, such as tapping, double tapping,

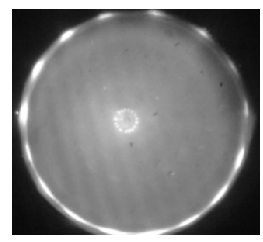

(a)

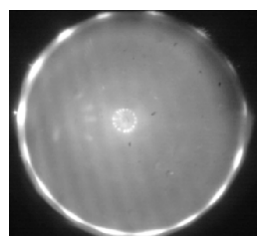

(b)

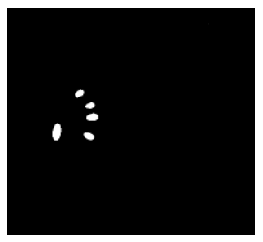

(c)
Figure 3: Finger tracking: (a) Background image (no fingers). The illumination is almost uniform on the sphere except close to the opening where we do not expect any tracking and at the pole where the LEDS are specularly reflected. (b) Sensed image with touching fingers. (c) Image after processing. dragging, flicking, pinching and others [12].

\subsection{Rendering and Calibration}

For the rendering and the calibration part, we rely on our own software based on $\mathrm{C}++$ and OpenGL shaders.

Projection The rendering consists in an azimuthal projection of a 2D image (see on the right). This projection needs to be calibrated once according to the hardware setup.

For this purpose, we use an interactive process (see on the right) that consist in: A) setting the center of the cross to the north pole of the sphere, so that the OpenGL position of the center of the images to

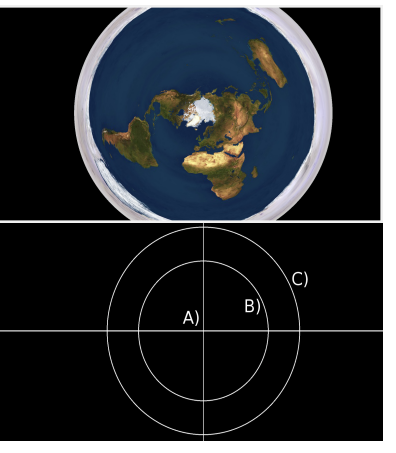
display is known. B) setting the middle circle on the equator of the sphere, so that a radial scaling can be applied to the image. C) setting the outside circle as the maximum visible circle on the sphere, so that a working area is defined where the tracking needs to be calibrated.

The circle defined in C) contains $55 \%$ of the total projected image which corresponds approximately to $760 \mathrm{k}$ pixels over the $1.4 \mathrm{M}$ pixels of the projector. In Section 4 , we propose a method to improve the final resolution. Note that the distribution of pixels over the sphere is not regular, as pixels close to the north pole are slightly bigger than pixels of low latitudes because of the high distorsion of the fisheye lens. Contrary to DLP projectors, there is no visible pixel grid and the image is continuous.

Tracking When the projection calibration is done, our tracking calibration algorithm aims at making a one to one correspondance between the projector's and camera's pixels. To do so, our calibration preprocess requires the user to touch a series of projected points (typically 35 points) on the sphere. The difference between projected points and detected points can be seen in Figure 4.

In order to extrapolate the mapping function to any pixel, a bilaplacian reconstruction is performed, with the sampled points as constraints and other pixels as unknowns.

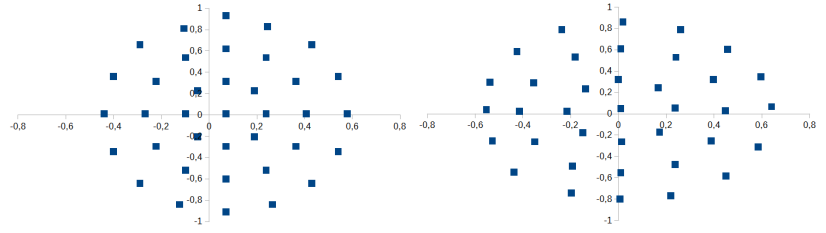

Figure 4: Tracking Calibration: A set of points (left) is projected. The user points them and the webcam senses their positions (right).

Correction of Chromatic Aberrations The optical system has some chromatic aberrations that degrade the image quality. Fortunately, the discrete spectrum of the projector allows a color correction by channel without any rainbow effect that would be induced by continuous light sources.

We correct the chromatic aberrations by applying the inverse color shifting to the input image. To do so, the user samples the color correction over the sphere by translating red and blue channels onto the green one (chosen as reference) at several points. As the aberrations are continuous, we 
perform in a preprocess, like the tracking calibration, a bilaplacian reconstruction to generate a color correction map.

Each image displayed on the sphere is first rendered into a frame buffer object, then a color correction shader is used on the GPU to correct the position of red and blue channels, thus minimizing color aberration effects.

\subsection{Prototype}

In order to keep the low-cost aspect of this work, all the lenses have been ordered from SurplusShed, a low-cost optics provider. Moreover, the mounts for the projector, lenses, cold mirror, and webcam are made by $3 \mathrm{D}$ printing. The different parts hold together thanks to $5 \mathrm{~mm}$ metallic rods and shaft collars.

As shown in Figure 1(a), the prototype holds vertically thanks to a homemade wood enclosure. The frosted glass sphere is part of lamp used for interior lighting, bought from the local hardware store. It has a diameter of $25 \mathrm{~cm}$ with and opening neck of $12 \mathrm{~cm}$, and is fixed at the top of the enclosure, just above the LED ring.

The total cost of this project is less than 500\$: the projector represents $379 \$$, the fisheye lens $35 \$$, the cold mirror $26 \$$, and the other components cost less than $15 \$$ each. In comparison, existing products such as Puffersphere [6] and Magic Planet [11], cost several thousands of dollars.

\section{Impact}

In this paper, we presented a more affordable spherical multitouch display than previous approaches. Its principal components are the projection through a fisheye lens via a laser-beam steering projector and optical finger tracking for multitouch. We offset the use of cheap material by software calibrations and corrections. An example of the flagship application of spherical multitouch applications, an interactive globe, can be seen in Figure 1(a).

The systems the most related to ours are the work from Benko et al. [2] and Patel [9]. Compared to Benko et al. [2], we only require off-the-shelf hardware components, and in particular, not a specifically tailored fisheye lens. Compared to Patel [9], our system is multitouch and, thanks to our optical system, covers the entire sphere.

We tested our spherical multitouch display with several users, experts as well as non-experts, that were enthusiastically able to use our system without any difficulty. To spread the use of our display and guide people willing to build their own, we intend to create a web site containing the software, details on the hardware setup as well as the $3 \mathrm{D}$ models of the printed components.

The main drawback of the proposed system is, as explained in Section 3.2, the lack of brightness that limits the use in bright environments. This is inherent to the projector which has only 30 lumens. As the development of LBS projectors is a growing trend, we expect this problem to be solved soon by replacing our projector by a future, brighter one.

\section{Future work}

Because of the shape of the lenses and the aspect ratio of the projector, the collected flux is a circular portion of the rectangular image that represents only $55 \%$ of the input image (see Section 3.3), which is as much loss in brightness and resolution. It is possible to use an anamorphic optical system, such as an anamorphic prism pair, to squeeze the image horizontally, from 16:9 aspect ratio to 1:1, in order to get up to $70 \%$ of the projector's beam.
Moreover, as mentioned in Section 3.3, the rear diffused illumination technique suffers from a lack of contrast, and the detection robustness could be improved. In 2005, Jeff Han [3] proposed the Frustrated Total Internal Reflection (FTIR) for low-cost finger tracking on planar multitouch displays. Infrared light, which is injected on the edges of the surface, propagates through the whole surface thanks to total internal reflection and escapes only where the fingers are in contact with the surface. As far as we know, the FTIR technique has never been used on spherical displays. From our experiments, the FTIR is compatible with spherical surfaces and presents a background image which is much darker, thus improving the tracking results.

However, an important issue has yet to be dealt with: for internal reflection to be efficient, the surface must be transparent, but we want it to be diffuse at the same time to be used as a projection surface. A solution would be to add a rear projection layer on the inside side of the sphere. We have tested the Rosco Grey projection layer, as proposed in [3], and it has shown good results in terms of projection quality and compatibility with FTIR. The problem lies, of course, in putting a planar layer on a spherical, yet non-developpable, surface.

We are investigating other solutions such as rear projection sprays, low refractive index coatings or distinct spheres for projection and tracking to adapt the FTIR technique to spherical displays.

\section{References}

[1] Hrvoje Benko and Andrew Wilson. Design challenges of interactive spherical user interfaces. In Proceedings of $\mathrm{CHI}$ 2009, 2009.

[2] Hrvoje Benko, Andrew D. Wilson, and Ravin Balakrishnan. Sphere: Multi-touch interactions on a spherical display. In Proceedings of UIST 2008, 2008.

[3] Jefferson Y. Han. Low-cost multi-touch sensing through frustrated total internal reflection. In Proceedings of UIST 2005, 2005.

[4] Martin Kaltenbrunner. http://tuio.org/?software, 2005. (Accessed on 11/30/2016).

[5] Thomas Ligon, Christopher Fedde, and Jonathan Lang. A self-contained spherical display system. In ACM Siggraph 2003 Emerging Technologies, 2003.

[6] Pufferfish Ltd. pufferfishdisplays.co.uk, 2002. (Accessed on $11 / 30 / 2016)$

[7] Christian Moore. nuigroup.com, 2006. (Accessed on 11/30/2016)

[8] Ye Pan, William Steptoe, and Anthony Steed. Comparing flat and spherical displays in a trust scenario in avatar-mediated interaction. In Proceedings of CHI 2014, 2014.

[9] Nirav Patel. Snow globe: Part one, cheap DIY spherical projection. pufferfishdisplays.co.uk, 2011. (Accessed on 11/30/2016).

[10] Peau Productions. https://www. peauproductions.com/, 2009. (Accessed on 11/30/2016).

[11] S.W. Utt, P.C. Rubesin, and M.A. Foody. Display system having a three-dimensional convex display surface, 2008. US Patent 7,352,340.

[12] Craig Villamor, Dan Willis, and Luke Wroblewski. Touch Gesture Reference Guide. (Accessed on 11/30/2016).

[13] Julie Williamson, Daniel Sunden, and Keith Hamilton. The lay of the land: Techniques for displaying discrete and continuous content on a spherical display. In PerDis '16: $A C M$ International Symposium on Pervasive Displays, 2016. 